BCCUNY-HEP/01-02

hep-th/0109041

\title{
Fundamental Strings and Cosmology
}

\author{
Ramzi R. Khuri* \\ Department of Natural Sciences, Baruch College, CUNY \\ 17 Lexington Avenue, New York, NY $10010^{\dagger}$ \\ Graduate School and University Center, CUNY \\ 365 5th Avenue, New York, NY 10036 \\ Center for Advanced Mathematical Sciences \\ American University of Beirut, Beirut, Lebanon **
}

\begin{abstract}
We show that the velocity-dependent forces between parallel fundamental strings moving apart in a $D$-dimensional spacetime imply an expanding universe in $D$-1-dimensional spacetime.
\end{abstract}

September 2001

* e-mail: khuri@gursey.baruch.cuny.edu. Research supported by a Eugene Lang Junior Faculty Research Fellowship.

$\dagger$ Permanent address.

** Associate member. 
In this paper, we show that the velocity-dependent forces between parallel fundamental strings in $D$ spacetime dimensions, with certain initial conditions, lead to an expanding universe in $D-1$ dimensions. While the model presented below is very simple, the result seems to be quite general and is expected to hold for more complicated scenarios. The findings seem consistent with recent observations [1] of an accelerating universe, with predictions for the late time expansion rate.

We start with the action $S=I_{D}+S_{2}$, where

$$
I_{D}=\frac{1}{2 \kappa^{2}} \int d^{D} x \sqrt{-g} e^{-2 \phi}\left(R+4(\partial \phi)^{2}-\frac{1}{12} H_{3}^{2}\right)
$$

is the $D$-dimensional string low-energy effective spacetime action and

$$
S_{2}=-\frac{\mu}{2} \int d^{2} \zeta\left(\sqrt{-\gamma} \gamma^{\mu \nu} \partial_{\mu} X^{M} \partial_{\nu} X^{N} g_{M N}+\epsilon^{\mu \nu} \partial_{\mu} X^{M} \partial_{\nu} X^{N} B_{M N}\right)
$$

is the two-dimesional worldsheet sigma-model action. Here $g_{M N}, B_{M N}$ and $\phi$ are the spacetime sigma-model metric, antisymmetric tensor and dilaton, respectively, while $\gamma_{\mu \nu}$ is the worldsheet metric. $H_{3}=d B_{2}$ and $\mu$ is the string tension.

The "fundamental string" (or "elementary" string) solution to the combined action, representing stationary macroscopic strings parallel to the $x^{1}$ direction, is given by [2]

$$
\begin{aligned}
d s^{2} & =h^{-1}\left(-d t^{2}+\left(d x^{1}\right)^{2}\right)+\delta_{i j} d x^{i} d x^{j}, \\
e^{-2 \phi} & =-B_{01}=h=1+\frac{k}{r^{n}},
\end{aligned}
$$

where $n=D-4, r^{2}=x^{i} x_{i}$ and the indices $i$ and $j$ run through the $D-2$-dimensional space transverse to the string. The constant $k$ is essentially the Noether charge of the source string and is proportional to its tension.

This solution can be extended to a multi-static string solution owing to the existence of a zero-force condition. This condition in turn arises from the cancellation between the attractive gravitational and dilatational forces of exchange with the repulsive antisymmetric field exchange, and is based on the existence of supersymmetry and the saturation of a BPS bound [3]. 
It was subsequently shown that, in addition to the zero static force, the leading order $\left(O\left(v^{2}\right)\right)$ velocity-dependent forces cancel for moving strings as well [4] (see also [5]). This result too is associated with the existence of higher supersymmetry [6].

Consider the Lagrangian for a test fundamental string moving in the background of another, source string. Replacing the fields of (3) in (2) leads to the Lagrandian

$$
\mathcal{L}=-\mu h^{-1}\left(\sqrt{1-h \dot{x}^{2}}-1\right)
$$

where $\dot{x}^{2}=\dot{x}^{i} \dot{x}_{i}$ and the "." represents a time derivative. The Euler-Lagrange equations for (四) lead via a straightforward calculation to

$$
\ddot{x}^{i}-\frac{n k \dot{x}^{2}\left(x^{j} \dot{x}_{j}\right) \dot{x}^{i}}{r^{n+2}\left(1-h \dot{x}^{2}\right)}+\frac{h\left(\dot{x}^{k} \ddot{x}_{k}\right) \dot{x}^{i}}{\left(1-h \dot{x}^{2}\right)}=\frac{\partial_{i} h}{h^{2}}\left(1-\frac{h \dot{x}^{2}}{2}-\sqrt{1-h \dot{x}^{2}}\right) .
$$

Contracting with $\dot{x}_{i}$ and simplifying leads to

$$
\dot{x}^{i} \ddot{x}_{i}=\frac{n k x^{j} \dot{x}_{j}}{2 h^{2} r^{n+2}}\left((y-1)^{2}(2 y+1)\right),
$$

where $y=\sqrt{1-h \dot{x}^{2}} \geq 0$. Since the term in parentheses in the right hand side of (6) is always positive, it follows that whenever $x^{j} \dot{x}_{j}>0$, it follows that $\frac{d}{d t}\left(\dot{x}^{2}\right)=2 \dot{x}^{i} \ddot{x}_{i}>0$. In other words, whenever the component of the relative velocity of the strings in the direction of their line of separation is parallel (as opposed to antiparallel) to the relative position vector, the relative speed is increasing. This means that the velocity-dependent force is repulsive whenever the strings are moving away from each other. This in turn has an interesting consequence for the separation distance between the strings.

It is straightforward to show from the Hamiltonian $H=\dot{x}^{i} p_{i}-\mathcal{L}$ following from (4) that the conserved energy of the two-string system is given by

$$
E=\frac{\mu}{h}\left(\frac{1}{\sqrt{1-h \dot{x}^{2}}}-1\right) .
$$

Setting $\dot{x}^{2}=u$, the function

$$
f(u, h)=\frac{1}{h}\left(\frac{1}{\sqrt{1-u h}}-1\right)=\frac{E}{\mu}=\rho
$$


is a constant. It is straightforward to show that $\partial f / \partial u>0$ and $\partial f / \partial h>0$ for all values of $u$ and $h$ (note that $u \leq 1, h>1$ and $u h<1$ ). Therefore, if $u=\dot{x}^{2}$ increases, as is the case above, then $h$ must decrease. This follows from requiring $d f=(\partial f / \partial u) d u+(\partial f / \partial h) d h=0$, so that if $d u>0$, then the positivity of the partial derivatives of $f(u, h)$ implies that $d h<0$. If $h=1+k / r^{n}$ decreases, it follows that $r$, the separation of the strings, must increase. Therefore if the strings are initially moving apart, the net velocity-dependent force between them is repulsive, which leads to a further separation of the strings. Since this type of interaction occurs for any two strings, if we start with any number of close, parallel strings initially moving apart in the transverse space, they will continue to do so indefinitely and will fuel an expanding universe in the $D$-2-dimensional transverse space and therefore in the $D$-1-dimensional spacetime orthogonal to the strings. For example, five-dimensional fundamental strings lead to an expanding universe in $D=4$ spacetime dimensions.

The time-dependence of the general expansion is also interesting. From (6), it is easy to see that the acceleration vector $\ddot{x}^{i}$ is always in the plane determined by the position vector $x^{i}$ and the velocity vector $\dot{x}^{i}$. It follows that the motion of the test-string in the two-body problem remains in the same plane. This allows us to use polar coordinates and write the Lagrangian as

$$
\mathcal{L}=-\mu h^{-1}\left(\sqrt{1-h\left(\dot{r}^{2}+r^{2} \dot{\theta}^{2}\right)}-1\right) .
$$

This Lagrangian has a conserved angular momentum

$$
L=\frac{\partial \mathcal{L}}{\partial \dot{\theta}}=\frac{\mu r^{2} \dot{\theta}}{\sqrt{1-h\left(\dot{r}^{2}+r^{2} \dot{\theta}^{2}\right)}}=l \mu .
$$

Solving for $\dot{x}^{2}$ from (『), one easily arrives at

$$
\dot{x}^{2}=\dot{r}^{2}+r^{2} \dot{\theta}^{2}=\frac{\rho(h \rho+2)}{(h \rho+1)^{2}} .
$$

Solving for $\dot{\theta}^{2}$ from (10) and replacing in (11) leads to

$$
\dot{r}^{2}=\frac{\rho(h \rho+2)\left(1+l^{2} h / r^{2}\right)}{(h \rho+1)^{2}\left(1+l^{2} / r^{2}\right)} .
$$


Now consider a universe consisting of many fundamental strings initially very close to each other. Each pair of such strings interacts as above, so that an initial outward propagation of the strings would tend to further push them apart in the transverse space. The exact solution of this problem requires a many-body computation, probably best done by numerical methods. For now, to obtain a rough idea as to the time dependence of the expansion of such a universe, let us assume that in a mean-field approximation, the effective force on each string may be approximated by that of a single, very large source fundamental string whose Noether charge $k$ is equal to the total charge of all of the strings in the $D$ dimensional space. In this approximation, the existence of nonzero angular momentum $l$ for the test string depends on the presence of an initial angular momentum in the universe. The distance $r$ between the test string and the source string in this model then represents the approximate average position of the strings, and hence the size of the universe. We wish to study the time dependence of $r$ at both early and late times for an expanding model.

In the very unlikely scenario of $l>>k^{1 / n}$, an immediate consequence of (12) is that the conditions $\dot{r} \leq 1$ and $1-\dot{x}^{2} h>0$ rule out this model for early times $r<<k^{1 / n}$. Furthermore, even with the more reasonable assumption of $l<<k^{1 / n}$, the model breaks down in the domain $r<<l$ for the same reasons. It is therefore reasonable to assume $l \simeq 0$ in the mean-field approximation, so that the general expansion can be regarded as essentially radial, i.e. the angular components of the velocity-dependent forces average to zero and $\dot{x}^{2} \simeq \dot{r}^{2}$. In any case, the existence of small nonzero $l$ does not greatly affect the qualitative conclusions regarding the expansion rate. In all domains, the strings are separating at an accelerated rate, with both $\dot{r}>0$ and $\ddot{r}>0$. For early times, $l<<r<k^{1 / n}$, with $r>>\left(k l^{2}\right)^{1 /(n+2)}$, the time-dependence of $r$ also depends on $n=D-4$, and hence on the dimension of the spacetime. For $n>2, r \sim(b-t)^{-1 /(n / 2-1)}$, where $b$ is a constant. The case of $n=2($ or $D=6)$ is the most interesting: $r \sim \exp (t / \sqrt{k})$, representing an exponential inflationary phase. For $n=1, r \sim t^{2}$. For all $n \geq 1$, the 
expansion proceeds with positive, outward acceleration. At very late times, for $r>>k^{1 / n}$, the expansion rate asymptotically approaches a constant speed $\dot{r} \rightarrow \sqrt{\rho(\rho+2) /(\rho+1)^{2}}$, again for all $n \geq 1$.

Without attaching too much importance to the exact quantitative predictions of this highly simplified model, one can still extract interesting consequences from this kind of string-seeded universe. The main feature is that, for certain initial conditions, the expansion is self-fueled by the repulsive velocity-dependent forces. This occurs because the initially separating strings lead to a repulsive force, which further separates the strings, etc. The exponentially expanding phase for $D=6$ is interesting, and suggests an inflationary phase. An interesting possibility in this case is that the moving strings in $D=6$ lead to an expanding five-dimensional universe, in which an effective four-dimensional brane universe resides, following [7]. The asymptotic late time expansion rate of $\sqrt{\rho(\rho+2) /(\rho+1)^{2}}$ is also intriguing, and may represent a testable prediction for this type of model. Further investigations of this type of model are clearly merited, using a possible combination of numerical computations, quantum string effects and nonequilibrium thermodynamics.

Note Added: After this paper appeared, it was pointed out to the author that a similar approach to string cosmology, though with somewhat different focus, was used in [8]. 


\section{References}

[1] A. G. Reiss et al., Astron. J. 116 (1998) 1009, astro-ph/9805201; S. Perlmutter et al., Astrophys. J. 517 (1999) 565, astro-ph/9812133.

[2] A. Dabholkar, G. W. Gibbons, J. A. Harvey and F. Ruiz Ruiz, Nucl. Phys. B340 (1990) 33.

[3] M. K. Prasad and C. M. Sommerfield, Phys. Rev. Lett. 35 (1975) 760; E. B. Bogomol'nyi, Sov. J. Nucl. Phys. 24 (1976) 449.

[4] C. G. Callan and R. R. Khuri, Phys. Lett. B261 (1991) 363.

[5] M. J. Duff, R. R. Khuri and J. X. Lu, Phys. Rep. B259 (1995) 213, hep-th/9412184.

[6] R. R. Khuri and R. C. Myers, Phys. Rev. D52 (1995) 6988, hep-th/9508045.

[7] L. J. Randall and R. Sundrum, Phys. Rev. Lett. 83 (1999) 3370; Phys. Rev. Lett. 83 (1999) 4690.

[8] A. Kehagias and E. Kiritsis, JHEP 9911 (1999) 022. 